Original Article

\title{
FORMULATION OPTIMIZATION AND EVALUATION OF FLURBIPROFEN EMULGEL
}

\author{
DIKSHA S. CHODANKAR ${ }^{*}$, SACHI S. KUDCHADKAR ${ }^{1}$, RAJASHREE S. GUDE², PRERANA D. NAVTI², SANAM M. \\ SAWANT ${ }^{1}$
}

${ }^{1}$ Department of Pharmaceutical Analysis, Goa College of Pharmacy, Panaji 403001, Goa, India, ${ }^{2}$ Department of Pharmaceutics, Goa College of Pharmacy, Panaji 403001, Goa-India

Email: dikshachodankar48@gmail.com

Received: 04 Mar 2020, Revised and Accepted: 06 Jun 2020

\section{ABSTRACT}

Objective: The objective of the present study was to formulate flurbiprofen (FLB) emulgel, evaluation of the formulations and the selection of an optimized formulation through in vitro drug release and drug content studies. Flurbiprofen is a non-steroidal anti-inflammatory drug (NSAID) requiring frequent administration and its chronic intake can lead to systemic side effects like gastric irritation and GI bleeding. The development of a dermal drug delivery system can overcome these side effects.

Methods: The emulgel formulations were produced using different combinations of oil and emulsifying agents. Carbopol 940 was used as a gelling agent. The prepared emulgels were evaluated for general appearance, $\mathrm{pH}$, spreadability, extrudability, drug content, in vitro drug release, average globule size and viscosity.

Results: Optimized formulation F7 showed a better in vitro drug release compared to the marketed gel preparation. The stability study for the optimized formulation was carried out at $25^{\circ} \mathrm{C} / 60 \% \mathrm{RH}$ for 3 mo and the emulgel was found to be stable concerning the physical appearance, pH and drug content.

Conclusion: The study revolved around the formulation of emulgel containing Flurbiprofen for dermal delivery of the drug. Emulgel was formulated with the purpose to enhance the permeation of poorly water-soluble drug FLB. The study concluded that the optimized emulge containing FLB exhibited better in vitro drug release profile compared to the marketed formulation.

Keywords: Flurbiprofen, Emulgel, Carbopol 940, Optimization, Evaluation

(C) 2020 The Authors. Published by Innovare Academic Sciences Pvt Ltd. This is an open access article under the CC BY license (http://creativecommons.org/licenses/by/4.0/) DOI: http://dx.doi.org/10.22159/ijpps.2020v12i8.37330. Journal homepage: https://innovareacademics.in/journals/index.php/ijpps.

\section{INTRODUCTION}

Non-Steroidal Anti-inflammatory Drugs (NSAIDs) are the category of drugs used for the long term treatment of Rheumatoid arthritis (RA) and osteoarthritis. When administered by oral route, these drugs cause systemic side effects like gastric ulceration and irritation but when administered as a dermal drug delivery system, the systemic side effects as well as the first-pass metabolism of the drug is bypassed thereby delivering the drug at a predetermined rate and improving patient compliance. Flurbiprofen, [2-(2-fluoro-4-biphenylyl) propionic acid] is a potent chiral non-steroidal anti-inflammatory agent with antipyretic and analgesic action and is approved by USFDA for the treatment of rheumatoid arthritis, osteoarthritis, and alkylosing spondylitis. It has a half-life of $4.7 \mathrm{~h}$ and needs frequent administration and its chronic intake could result in systemic side effects like gastric irritation and gastric bleeding [1-5].

Owing to the various disadvantages associated with semisolids like ointments, creams and lotions like stickiness, less spreading abilities and the need for the application of semisolids with rubbing and stability issues, transparent gels are more preferred in cosmetics as well as pharmaceutical preparations but the drawback of gels is the inability to incorporate and deliver hydrophobic drugs through the aqueous gel base. To overcome this disadvantage, a novel emulgel approach could be used to incorporate the hydrophobic drug into the aqueous gel base. Emulgels are either oil in water or water in oil emulsions gelled by mixing with a gelling agent and combine the properties of both gel and emulsions thereby acting as a dual control release systems [6-7]

The purpose of this novel work was to formulate flurbiprofen emulgel, providing therapeutic effect at the localized site and eliminating gastrointestinal side effects associated with the oral formulations. The emulgel was formulated using Carbomer 940 as a polymer. The effect of varying concentration of emulsifying agents and oil phase on the drug release was investigated.

\section{MATERIALS AND METHODS}

Flurbiprofen was obtained as a gift sample from FDC-Ltd (Roha Maharashtra), carbopol 940 was obtained from lubrizol, tween 80 span 80 and triethanolamine were obtained from molychem, liquid paraffin was obtained from SD-chemicals and fines ltd. All the solvents were of analytical grade.

Preparation of an emulgel

The composition of an emulgel is as shown in table I.

Preparation of Carbopol 940 gel base

Carbopol 940 was soaked in a sufficient quantity of distilled water for $3 \mathrm{~h}$ and then $\mathrm{pH}$ was adjusted to 6.5 using triethanolamine to yield the gel.

\section{Preparation of an emulsion}

Preparation of oil phase: Oil phase of the emulsion was prepared by dissolving Span 80 in light liquid paraffin followed by dispersing the drug in it since Flurbiprofen is hydrophobic by nature.

Preparation of an aqueous phase: The aqueous phase was prepared by dissolving Tween 80 in purified water. Methylparaben was then dissolved in a sufficient quantity of ethanol and propylene glycol and added to the aqueous phase. Both the oily and aqueous phases were separately heated to $70-80^{\circ} \mathrm{C}$, then the oily phase was added to the aqueous phase with continuous stirring followed by cooling to room temperature to yield an o/w emulsion.

\section{Preparation of an emulgel}

The final emulgel was formed by the addition of the emulsion to Carbopol gel in the ratio of 1:1 with constant stirring [8]. 
Table 1: Composition of different formulation batches $(\% \mathrm{w} / \mathrm{w})$

\begin{tabular}{|c|c|c|c|c|c|c|c|c|c|}
\hline Formula & F1 & F2 & F3 & F4 & F5 & F6 & F7 & F8 & F9 \\
\hline Flurbiprofen & 1 & 1 & 1 & 1 & 1 & 1 & 1 & 1 & 1 \\
\hline Carbopol 940 & 1 & 1 & 1 & 1 & 1 & 1 & 1 & 1 & 1 \\
\hline Liquid paraffin & 5 & 5 & 5 & 7.5 & 7.5 & 7.5 & 10 & 10 & 10 \\
\hline Span 80 & 0.9 & 1.9 & 2.8 & 0.9 & 1.9 & 2.8 & 0.9 & 1.9 & 2.8 \\
\hline Tween 80 & 1.1 & 2.1 & 3.2 & 1.1 & 2.1 & 3.2 & 1.1 & 2.1 & 3.2 \\
\hline Propylene glycol & 5 & 5 & 5 & 5 & 5 & 5 & 5 & 5 & 5 \\
\hline Ethanol & 2 & 2 & 2 & 2 & 2 & 2 & 2 & 2 & 2 \\
\hline Methyl Paraben & 0.03 & 0.03 & 0.03 & 0.03 & 0.03 & 0.03 & 0.03 & 0.03 & 0.03 \\
\hline Purified water & \multirow{2}{*}{\multicolumn{9}{|c|}{$\begin{array}{l}\text { qs to } 100 \mathrm{ml} \\
\text { qs to adjust pH to } 6 \text { to } 7\end{array}$}} \\
\hline Triethanolamine & & & & & & & & & \\
\hline
\end{tabular}

\section{Drug-polymer compatibility study}

The drug-polymer compatibility study was carried out using FTIR spectrophotometer (Schimadzu). The IR spectra of the drug as well as the physical mixture of the drug and polymer in the ratio of $1: 1$ were recorded. All the spectras were recorded in the range of 400 $4000 \mathrm{~cm}^{-1}[8]$.

\section{Characterization of the formulated emulgel}

\section{General appearance}

The prepared emulgel formulations were visually inspected for color and appearance since no quantitative estimation could be done on this parameter due to the non-availability of the required facility.

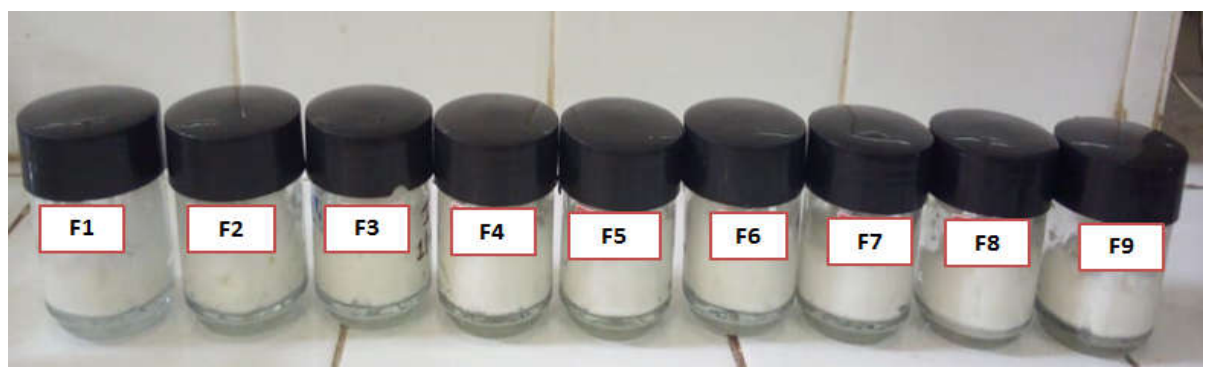

Fig. 2: Formulated emulgels

\section{pH}

The pH of the skin is normally between 6 to 7 , and it is therefore desirable that the preparation used for topical application does not fall beyond this value or else it will cause severe irritation to the skin. The $\mathrm{pH}$ of all the formulations was evaluated using a $\mathrm{pH}$ meter and the $\mathrm{pH}$ was measured at room temperature [8].

\section{Spreadability}

To study the spreadability of formulations, a special apparatus was designed. Spreadability was expressed in terms of time in seconds taken by two slides to slip off from formulations placed between, under the application of a certain load. Lesser the time taken to separate the two slides, better is the spreadability. Two glass slides of $6 \times 2 \mathrm{~cm}$ each were selected. The formulation was placed over one of the slides. This slide was placed on another slide in such a way that the formulation was sandwiched between the two slides. The formulation between the two slides was squeezed consistently to frame a slight layer; for this reason, weight $100 \mathrm{~g}$ was placed on the upper slide. The excess of the formulation adhering to the slide was scrapped off after the weight was removed. The lower slide was fixed on the surface of the apparatus and the upper slide was tied to the string. To this string, $20 \mathrm{~g}$ load was applied with the help of a simple pulley. Under the direction of weight applied, the time taken for the upper slide to move the distance i.e. of $6 \mathrm{~cm}$ and separate away from the other slide (lower) was noted. The experiment was repeated three times and the average of such determination was calculated for each formulation by using the formula $[9,10]$ :

$$
\mathrm{S}=\mathrm{M} \times \mathrm{L} / \mathrm{T}
$$

Where, $\mathrm{S}=$ spreadability,

$\mathrm{M}=$ weight tied to the upper slide,

$\mathrm{L}=$ length of glass slides
$\mathrm{T}=$ time taken to separate the slides completely from each other

\section{Extrudability}

Test to measure the force required to expel the material from the tube. The formulation whose extrudability was to be checked was filled in a clean, lacquered aluminium collapsible metal tube. Extrudability of the emulgel was the weight required to extrude from a lacquered aluminum collapsible tube at least $0.5 \mathrm{~cm}$ ribbon of emulgel in $10 \mathrm{~s}$ on the application of weight in grams. More the quantity extruded better is the extrudability. The measurement of the extrudability of each formulation was determined in triplicate and the average values are presented. The extrudability is than calculated by using the following formula [11]:

Extrudability $=$ Applied weight to extrude emulgel from tube (in g) /Area $\left(\right.$ in $\left.\mathrm{cm}^{2}\right)$

\section{Drug content}

Gel formulation (1 gram) was dissolved in methanol. The solution was filtered to obtain a clear solution. The absorbance of the resulting solution was measured using a UV Visible spectrophotometer after suitable dilution to determine the drug content [12].

\section{In vitro drug release}

In vitro release studies were done using diffusion studies. In vitro drug release behavior of the drug from the emulgel formulations was investigated using the dialysis membrane. Dialysis membrane previously soaked in the phosphate buffer $\mathrm{pH} 7.4$ was used as the permeation membrane. $50 \mathrm{ml}$ of Phosphate buffer $\mathrm{pH} 7.4$ was placed in a beaker (receptor compartment). An accurately weighed quantity $(1 \mathrm{~g})$ of the formulated emulgel was then uniformly spread on the dialysis membrane (donor compartment) and this membrane was tied to the diffusion tube (a hollow tube open on both sides). One 
side of the dialysis membrane was kept in contact with the medium phosphate buffer $\mathrm{pH}$ 7.4. The medium was constantly agitated using a magnetic stirrer and the temperature was maintained at a constant of $37 \pm 1{ }^{\circ} \mathrm{C}$ throughout the operation. Samples of $5 \mathrm{ml}$ volume were then withdrawn from the receptor compartment at intervals of 1 hour for $8 \mathrm{~h}$ and the amount withdrawn was replaced with a fresh volume of the medium. The samples withdrawn were then analyzed for the amount of Flurbiprofen released by UV spectrophotometric method by measuring the absorbance of the samples at $247 \mathrm{~nm}$ against Phosphate Buffer pH 7.4 taken as blank [8]

Table 2: Details of in vitro release studies

\begin{tabular}{ll}
\hline Diffusion medium & pH 7.4 Phosphate buffer \\
\hline Volume of diffusion medium used & $50 \mathrm{ml}$ \\
Temperature & $37^{\circ} \mathrm{C} \pm 1{ }^{\circ} \mathrm{C}$ \\
Time interval for sampling & $1 \mathrm{~h}$ \\
Speed & $50 \mathrm{rpm}$ \\
Volume of sample withdrawn & $5 \mathrm{ml}$ \\
Detection wavelength & $247 \mathrm{~nm}$ \\
\hline
\end{tabular}

\section{Average globule size}

The average globule size was measured by a light microscope at $40 \mathrm{X}$ magnification [8]

\section{Viscosity}

The viscosity of the optimized emulgel formulations is determined by Brookfield Viscometer using spindle no. 64 at 10 rpm [5].

\section{Stability study}

Stability study was performed on the optimized formulation F7. The preparation was packed in an aluminum collapsible tube $(5 \mathrm{~g})$ and was subjected to stability studies as $25{ }^{\circ} \mathrm{C} / 60 \% \mathrm{RH}$ for $3 \mathrm{mo}$.
Thereafter the samples were withdrawn and evaluated for physical appearance, rheological properties, and drug content. All the test results were found to be in limits. Hence the formulations were stable understated storage conditions $[6,13,16]$.

\section{RESULTS}

\section{Drug-polymer compatibility study}

The FTIR spectra of the pure Flurbiprofen and 1:1 mixture of Flurbiprofen and carbopol $940 \mathrm{P}$ is shown in fig. 2 and fig. 4 , respectively. There was no change in the position or disappearance of any characteristic peak of Flurbiprofen, indicating the compatibility between the drug Flurbiprofen and polymer carbopol 940

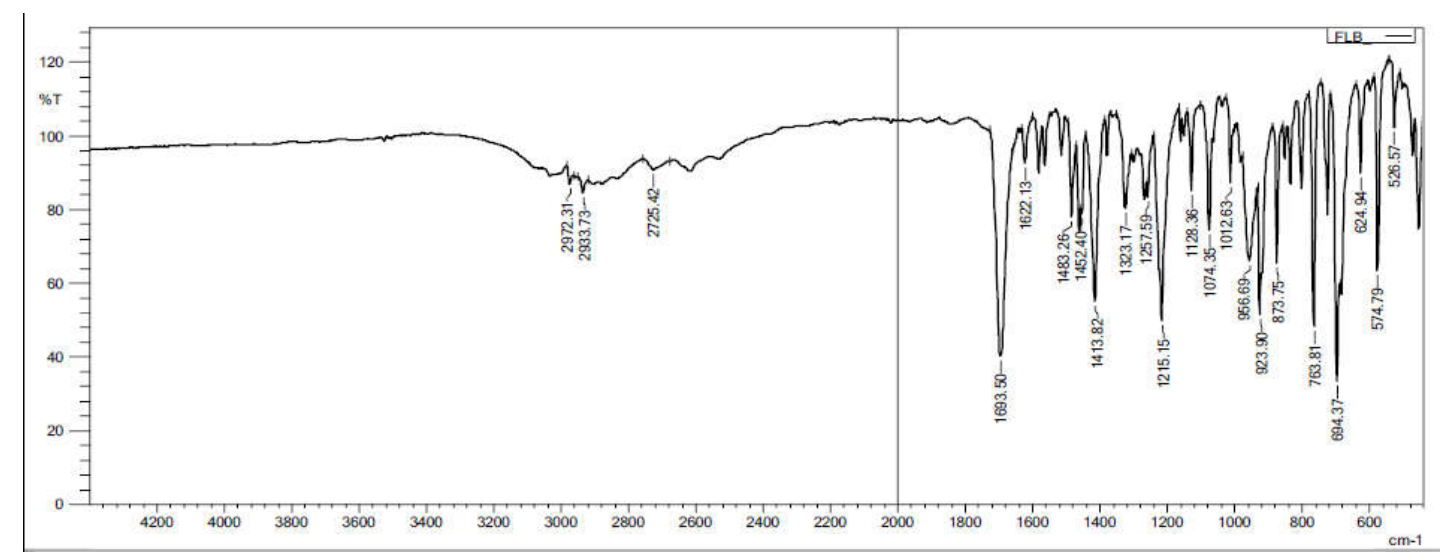

Fig. 2: FTIR spectra of flurbiprofen

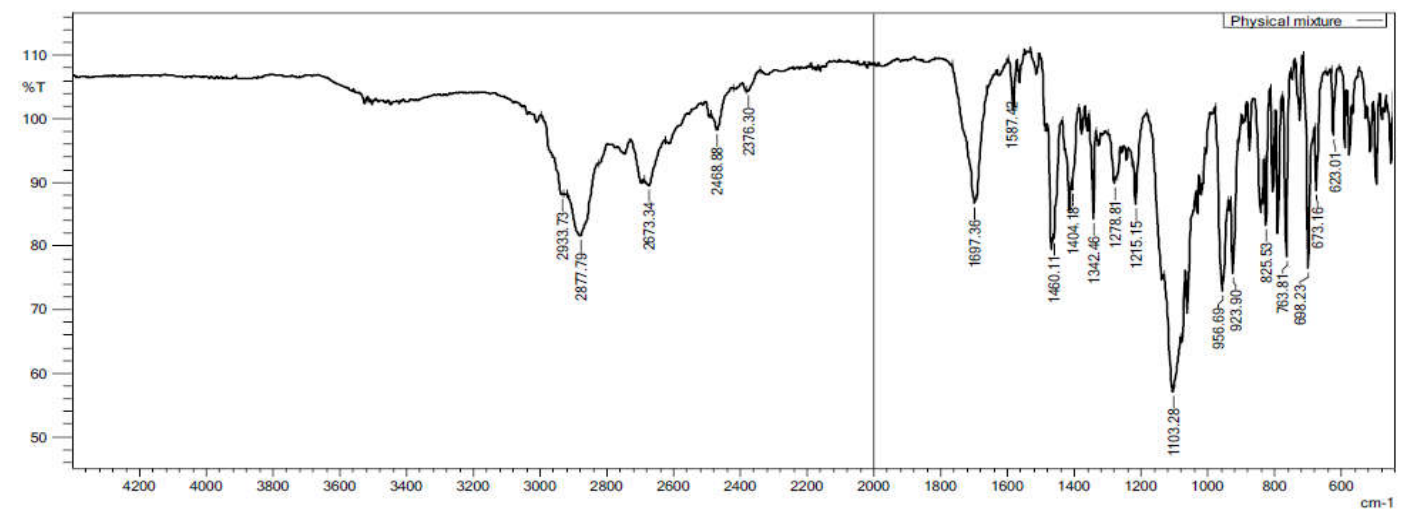

Fig. 3: FTIR spectra of 1:1 mixture of flurbiprofen and carbopol 940 


\section{General appearance}

All formulation batches were found to be homogenous yellowish milky emulsions while emulgels were found to be yellowish-white viscous, creamy preparations.

pH

The $\mathrm{pH}$ of all the formulations was found to be as in table 3 follows

\section{Spreadability}

The spreadability of all the formulations ranged as follows, as in table 4. Formulation F7 was found to possess the highest spreadability.

Drug content

$\%$. Drug content of all the formulations is as follows in table 5.

Table 3: pH of formulations F1-F9

\begin{tabular}{ll}
\hline Formulation code & ${ }^{*} \mathbf{p H}$ \\
\hline F1 & $6.2 \pm 0.76$ \\
F2 & $6.4 \pm 0.88$ \\
F3 & $6.7 \pm 0.79$ \\
F4 & $6.1 \pm 0.66$ \\
F5 & $6.2 \pm 0.50$ \\
F6 & $6.8 \pm 0.77$ \\
F7 & $6.7 \pm 0.87$ \\
F8 & $6.3 \pm 0.99$ \\
F9 & $6.8 \pm 0.95$ \\
\hline
\end{tabular}

*Data are represented as mean \pm standard deviation (SD), $\mathrm{n}=3$

Table 4: Spreadability of formulations F1-F9

\begin{tabular}{ll}
\hline Formulation code & Spreadability value (cms) \\
\hline F1 & 6.66 \\
F2 & 5.83 \\
F3 & 6.66 \\
F4 & 7.66 \\
F5 & 7.00 \\
F6 & 6.83 \\
F7 & 7.97 \\
F8 & 7.33 \\
F9 & 7.33 \\
\hline
\end{tabular}

Table 5: Drug content of formulations F1-F9

\begin{tabular}{ll}
\hline Formulation code & ${ }^{*}$ Drug content $(\%)(\mathbf{m e a n} \pm \mathbf{S D}, \mathbf{n = 3})$ \\
\hline F1 & $98.90 \pm 0.73$ \\
F2 & $99.50 \pm 1.54$ \\
F3 & $99.80 \pm 2.59$ \\
F4 & $99.40 \pm 0.78$ \\
F5 & $98.42 \pm 0.98$ \\
F6 & $97.93 \pm 0.89$ \\
F7 & $100.0 \pm 0.99$ \\
F8 & $98.32 \pm 1.65$ \\
F9 & $99.42 \pm 0.84$ \\
\hline
\end{tabular}

*Data are represented as mean \pm standard deviation (SD), $n=3$

Table 6: Average droplet size range for formulations F1-F9

\begin{tabular}{ll}
\hline Formulation code & ${ }^{*}$ Average globule size $(\boldsymbol{\mu m})(\mathbf{m e a n} \pm \mathbf{S D}, \mathbf{n}=\mathbf{3})$ \\
\hline F1 & $14.5 \pm 0.25$ \\
F2 & $13.5 \pm 0.75$ \\
F3 & $11.5 \pm 1.00$ \\
F4 & $17.5 \pm 0.25$ \\
F5 & $17.0 \pm 0.95$ \\
F6 & $13.1 \pm 0.25$ \\
F7 & $14.0 \pm 1.25$ \\
F8 & $12.4 \pm 1.25$ \\
F9 & $16.7 \pm 0.95$ \\
\hline
\end{tabular}

*Data are represented as mean \pm standard deviation (SD), $n=3$

\section{Average droplet size}

The average droplet size measurements are as shown in the table 6.

\section{In vitro drug release}

The In vitro drug release of all the formulations F1-F9 and marketed gel formulation ranged as follows. 
Table 7: In vitro drug release of formulations F1-F9 and marketed gel formulation

\begin{tabular}{|c|c|c|c|c|c|c|c|c|c|c|}
\hline \multirow[t]{2}{*}{ Time (h) } & \multicolumn{10}{|c|}{$\%$ Cumulative drug release (\%CDR) } \\
\hline & F1 & F2 & F3 & F4 & F5 & F6 & F7 & F8 & F9 & Marketed gel formulation \\
\hline 0 & 0 & 0 & 0 & 0 & 0 & 0 & 0 & 0 & 0 & 0 \\
\hline 1 & 19.77 & 15.39 & 13.53 & 17.32 & 22.16 & 13.51 & 26.59 & 22.00 & 20.59 & 6.73 \\
\hline 2 & 30.14 & 29.68 & 21.74 & 29.30 & 32.16 & 23.88 & 42.71 & 40.42 & 38.71 & 10.73 \\
\hline 3 & 41.41 & 37.44 & 35.25 & 45.44 & 43.14 & 30.99 & 57.40 & 55.98 & 50.40 & 15.20 \\
\hline 4 & 44.52 & 42.08 & 39.76 & 53.90 & 51.57 & 34.84 & 68.72 & 65.47 & 62.88 & 23.54 \\
\hline 5 & 51.30 & 48.48 & 42.90 & 60.44 & 56.57 & 40.31 & 79.59 & 78.28 & 70.90 & 26.22 \\
\hline 6 & 58.98 & 55.25 & 52.88 & 63.54 & 60.11 & 50.31 & 88.42 & 83.09 & 81.44 & 30.32 \\
\hline 7 & 64.74 & 63.67 & 58.96 & 69.88 & 66.39 & 57.06 & 97.97 & 93.80 & 90.54 & 34.17 \\
\hline 8 & 69.23 & 66.91 & 63.26 & 74.10 & 71.59 & 62.90 & 99.00 & 95.18 & 92.67 & 38.90 \\
\hline
\end{tabular}

Table 8: Values of the regression coefficient $\left(R^{2}\right)$ and release kinetics of formulation in phosphate buffer pH 7.4

\begin{tabular}{llllllll}
\hline Code & Zero-order & \multicolumn{3}{l}{ First-order } & Peppas & Higuchi \\
\cline { 2 - 7 } & $\mathbf{R}^{\mathbf{2}}$ & $\mathbf{K}$ & $\mathbf{R}^{\mathbf{2}}$ & $\mathbf{K}$ & $\mathbf{R}^{\mathbf{2}}$ & $\mathbf{K}$ & $\mathbf{R}^{\mathbf{2}}$ \\
\hline F1 & 0.9485 & 7.99 & 0.9672 & 0.287 & 0.994 & 0.59 & 0.994 \\
F2 & 0.9599 & 7.91 & 0.9416 & 0.323 & 0.985 & 0.68 & 0.991 \\
F3 & 0.9712 & 7.65 & 0.9540 & 0.357 & 0.987 & 0.75 & 0.987 \\
F4 & 0.9341 & 8.96 & 0.9160 & 0.331 & 0.977 & 0.70 & 0.983 \\
F5 & 0.9278 & 8.13 & 0.890 & 0.0668 & 0.994 & 0.56 & 0.994 \\
F6 & 0.9815 & 7.40 & 0.974 & 0.346 & 0.991 & 0.71 & 0.977 \\
F7 & 0.967 & 10.56 & 0.956 & 0.310 & 0.995 & 0.65 & 0.994 \\
F8 & 0.955 & 10.39 & 0.930 & 0.335 & 0.985 & 0.70 & 41.75 \\
F9 & 0.970 & 10.23 & 0.943 & 0.343 & 0.991 & 0.72 & 0.991 \\
\hline
\end{tabular}

Table 9: The extrudability values of formulations F1-F9

\begin{tabular}{ll}
\hline Formulation code & ${ }^{*}$ Extrudability $\left(\mathbf{g} / \mathbf{c m}^{2}\right)(\mathbf{m e a n} \pm \mathbf{S D}, \mathbf{n}=\mathbf{3})$ \\
\hline F1 & $9.0 \pm 0.15$ \\
F2 & $7.5 \pm 0.4$ \\
F3 & $6.9 \pm 0.9$ \\
F4 & $8.5 \pm 1.0$ \\
F5 & $7.3 \pm 1.5$ \\
F6 & $8.0 \pm 0.25$ \\
F7 & $10.0 \pm 0.30$ \\
F8 & $7.0 \pm 1.5$ \\
F9 & $7.2 \pm 0.25$ \\
\hline
\end{tabular}

*Data are represented as mean \pm standard deviation (SD), $n=3$

Table 10: Results of the stability study (mean $\pm S D, n=3$ )

\begin{tabular}{|c|c|c|c|c|c|}
\hline Before & & & After & & \\
\hline Appearance & $* \mathrm{pH}$ & *Drug content (\%) & Appearance & ${ }^{*} \mathrm{pH}$ & *Drug content $(\%)$ \\
\hline White, creamy, viscous & $6.7 \pm 0.53$ & $100 \pm 1.53$ & White, creamy, viscous & $6.6 \pm 0.73$ & $98 \pm 1.00$ \\
\hline
\end{tabular}

\section{Extrudability}

The extrudability values of formulations F1-F9 were found to be as per table 9 .

\section{Viscosity}

Viscosity measurement was performed on the optimized formulation (F7) and was found to be $36300 \mathrm{cPs}$.

\section{Stability studies}

The results of the stability studies are as follows in table 10 .

\section{DISCUSSION}

FTIR was performed to detect any sign of interaction, which would be reflected by a change in the position or disappearance of any characteristic peak of Flurbiprofen. IR scans of pure drug flurbiprofen and 1:1 physical mixtures of flurbiprofen and carbopol 940 were taken. From the IR spectras shown in fig. 2 and fig. 3, it was observed that there was no interaction of the drug with any of the excipients. The appearance of the formulations was found to be satisfactory. The $\mathrm{pH}$ of all the formulations was found to be neutral and was close to the $\mathrm{pH}$ of the skin and hence it should not cause any irritation to the skin [14].

The spreadability of all the formulations was satisfactory. The highest spreadability was shown by optimized formulation F7 ie $7.97 \mathrm{~cm}$. Drug content was almost uniform in all the formulations and was found to be between 97.9 to $100.8 \%$. The results of the average globule size indicate the globule size of droplets varied from 11.5 to $17.5 \mu \mathrm{m}$. The in vitro release of the drug from the formulated emulgels was found to be higher as compared to the marketed Brufen gel. Optimized formulation F7 showed a release of 99\%, whereas the marketed formulation showed a release of $38.90 \%$ indicating that the formulated emulgel had better release compared to the marketed product. The release of the drug from the emulgel formulations could be ranked in the descending order as follows: F $7>$ F $8>$ F $9>$ F $4>$ F $5>$ F $6>$ F $1>$ F $2>$ F 3

The release was found to vary according to the concentration of liquid paraffin, span 80 and tween 80 . When the concentration of emulsifier's i. e span 80 and tween 80 was less; the release exhibited was more.

The in vitro release data was fitted to the curve kinetic models to know the mechanism of drug release. Regression coefficient $\left(R^{2}\right)$ 
values for zero-order ranged from 0.927-0.981 and for first-order plots ranged from $0.890-0.974$. $\mathrm{R}^{2}$ values were found to be higher for zero-order than for first-order. According to the regression coefficients tabulated in table 8 for all the formulations, it is evident that all the formulations follow zero-order drug release kinetics. Since regression coefficients of higuchi plot were found to be close to 1 according to the above-tabulated data, it also reveals that all the formulations exhibit diffusion drug release mechanism. In the case of Korsemeyer peppas plot ' $\mathrm{n}$ ' values were more than 0.5 , which indicates non-fickian drug release kinetics. Hence, from the data obtained from all the models, it was concluded that the drug release through the flurbiprofen emulgel formulations is diffusioncontrolled following zero-order kinetics with non-fickian diffusion pattern $[11,18]$.

The viscosity of the optimized formulation was found to be satisfactory.

The results of the stability studies indicated that the optimized emulgel was found to be stable concerning to physical appearance, $\mathrm{pH}$ and drug content at $25{ }^{\circ} \mathrm{C} / 60 \% \mathrm{RH}$ for a period of 3 mo.

\section{CONCLUSION}

The present study deals with the formulation development and optimization of flurbiprofen emulgel. The optimization was done based upon the drug content and in vitro drug release. The kinetic modeling revealed that Flurbiprofen was a good fit to the zero-order and Higuchi model. The emulgel was found to exhibit diffusion controlled drug release according to Higuchi model. Overall the release from the emulgel was zero-order, non-fickian drug release. Formulation batch F7 showed better drug release compared to the marketed gel (Brufen) formulation thus revealing the better results of the formulated emulgel. Thus the successful attempt of emulgel formulation was made.

\section{FUNDING}

$\mathrm{Nil}$

\section{AUTHORS CONTRIBUTIONS}

The research idea was set with the guidance of Prof. Sachi S. Kudchadkar. The research work was done by Diksha S. Chodankar. The drug was provided by Prof. Rajashree S. Gude. Calculations and interpretations were supported by Prerana D. Navti and Sanam M. Sawant. All the assistance in manuscript preparation and revision was provided by Prerana D. Navti.

\section{CONFLICT OF INTERESTS}

Declared none

\section{REFERENCES}

1. Wongrakpanich S, Wongrakpanich A, Melhado K, Rangaswami J. A comprehensive review of non-Steroidal anti-inflammatory drug use in the elderly. Aging Dis 2018;9:143-50.

2. Rudrangi S, Kaialy W, Ghori M, Trivedi V, Snowden M, Alexander B. Solid-state flurbiprofen and methyl- $\beta$ cyclodextrin inclusion complexes prepared using a single-step, organic solvent-free supercritical fluid process. Eur J Pharm Biopharm 2016;104:164-70.

3. Paliwal S, Tilak A, Sharma J, Dave V, Sharma S, Verma K. et al. Flurbiprofen-loaded ethanolic liposome particles for biomedical applications. J Microbiol Methods 2019;161:18-27.

4. Tiwari R, Tiwari G. Dissolution is modulating mechanism of flurbiprofen solid dispersions: characterization, physical stability and in vivo performance: formulation considerations and optimization study. Pharm Methods 2017;8:127-35.

5. Sarfaraz M, Sharma S. Formulation and evaluation of flurbiprofen fast disintegrating tablets using natural disintegrants. Asian J Pharm Clin Res 2016;9:247-54.

6. Redkar M, Patil S, Rukari T. Emulgel: a modern tool for topical drug delivery. World J Pharm Res 2019;8:586-97.

7. Ajazuddin, Alexander A, Khichariya A, Gupta S, Patel R, Giri T, et al. Recent expansions in an emergent novel drug delivery technology: Emulgel. J Controlled Release 2013;171:122-32.

8. Khunt D, Mishra A, Shah D. Formulation design and development of piroxicam emulgel. Int J PharmTech Res 2012;4:1332-44

9. Bhanu VP, Shanmugam V, Lakshmi PK. Development and optimization of novel diclofenac emulgel for topical drug delivery. Int J Contemp Pediatr 2020;9:1-4.

10. Bhattacharya S, Prajapati B. Formulation and optimization of celcoxib nanoemulgel. Asian J Pharm Clin Res 2017;10:353-65.

11. Joshi Baibhav, Singh Gurpreet, Rana AC, Saini Seema. Development and characterization of clarithromycin emulgel for topical delivery. Int J Drug Dev Res 2012;4:310-23.

12. Jain A, Gautam S, Gupta Y, Khambete H, Jain S. Development and characterization of ketoconazole emulgel for topical drug delivery. Der Pharm Sin 2010;1:221-31.

13. MR, VS, RN, KM. Formulation and in vitro evaluation of ciprofloxacin loaded topical emulgel. Int J Pharmacol Clin Sc 2016;1:237-41.

14. Sah S, Badola A, Mukhopadhyay S. Development and evaluation of tioconazole loaded emulgel. Int J Appl Pharm 2017;9:83-90.

15. Vani Y, Reddy C. Formulation and in vitro evaluation of piroxicam emulgel. Int J Pharm Sci Drug Res 2018;10:227-32.

16. Mohamed M. Optimization of chlorphenesin emulgel formulation. AAPS J 2004;6:81-7. 